Supporting information for

\title{
Structural Analysis and Identification of Colloidal Aggregators in Drug Discovery
}

Zi-Yi Yang ${ }^{1}$, Zhi-Jiang Yang ${ }^{1}$, Jie Dong ${ }^{2}$, Liang-Liang Wang ${ }^{3}$, Liu-Xia Zhang ${ }^{1}$, Jun-Jie Ding ${ }^{3}$, Xiao-Qin Ding ${ }^{3}$, Ai-Ping Lu ${ }^{4}$, Ting-Jun Hou ${ }^{5, *}$, Dong-Sheng Cao ${ }^{1,4, *}$

${ }^{1}$ Xiangya School of Pharmaceutical Sciences, Central South University, Changsha, 410003, P.R. China.

${ }^{2}$ Central South University of Forestry and Technology, Changsha, 410004, P.R. China

${ }^{3}$ Beijing Institute of Pharmaceutical Chemistry, Beijing 102205, P. R. China

${ }^{4}$ Institute for Advancing Translational Medicine in Bone and Joint Diseases, School of Chinese Medicine, Hong Kong Baptist University, Hong Kong SAR, China.

${ }^{5}$ College of Pharmaceutical Sciences, Zhejiang University, Hangzhou 310058, Zhejiang, P. R. China

\section{Corresponding authors}

Dongsheng Cao

Email: oriental-cds@163.com

Tel: $+86-731-89824761$

\section{Tingjun Hou}

E-mail: tingjunhou@zju.edu.cn

Tel: +86-571-88208412 

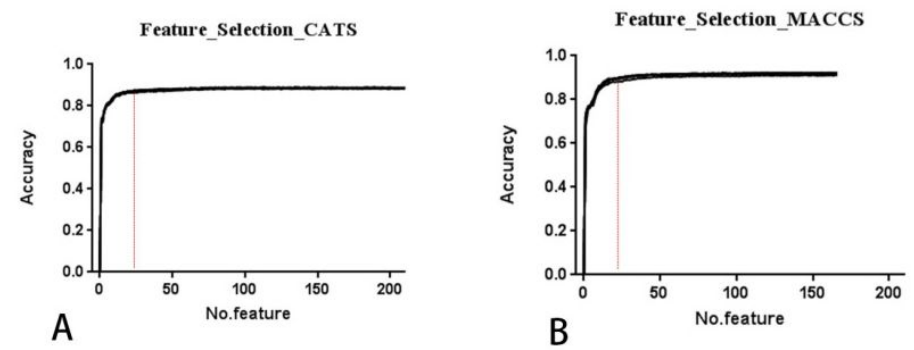

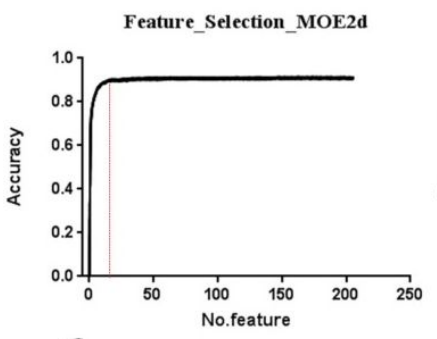

C

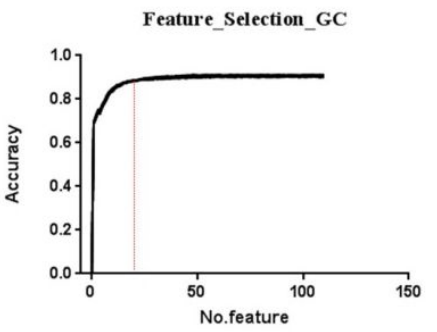

D

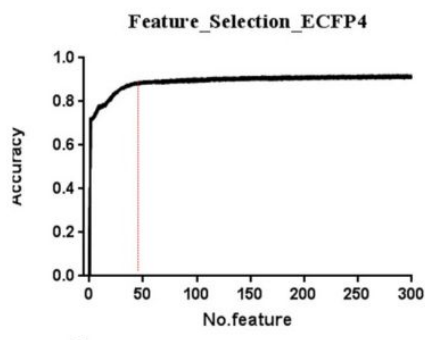

E

Figure S1. The feature selection results of five descriptors. A: CATS; B: MACCS; C: MOE2d; D: GC; E: ECFP4. 